\title{
Is osteoporosis risk in anorexia nervosa underestimated? A case report series
}

\author{
Bruno Pacciardi, Claudio Cargioli, Simone Belli, Biagio Cotugno, Samanta Augusto, Luca Di Paolo, \\ Mauro Mauri
}

Clinical and Experimental Medicine Department (Psychiatry Division), University of Pisa, Italy

Email: mauro.mauri@med.unipi.it

Received 7 January 2013; revised 7 February 2013; accepted 14 February 2013

Copyright (C) 2013 Bruno Pacciardi et al. This is an open access article distributed under the Creative Commons Attribution License, which permits unrestricted use, distribution, and reproduction in any medium, provided the original work is properly cited.

\begin{abstract}
Introduction: Anorexia nervosa (AN) is a mental disorder whose features are deliberate weight loss, disordered body image, and intrusive overvalued fears of gaining weight. Long-term consequences of AN include endocrine dysfunctions leading to secondary amenorrhea, bone loss and/or osteoporosis with an increased risk of bone fracture. Therefore young women with AN may develop a risk for bone fractures comparable to that of postmenopausal women. Methods: In this case report series Bone Mineral Density (BMD) was examined by Dual energy X-ray Absorptiometry (DXA) in 19 hospitalized patients with diagnosis of AN and prolonged amenorrhea. Results: All patients showed a lumbar/femoral bone loss or osteoporosis, with an increased fracture risk comparable to that of postmenopausal women. Conclusions: Our observation suggests that DXA evaluation of anorexic patients with prolonged amenorrhea would be helpful to prevent fracture risk in this population of patients. However, although DXA is almost routinely recommended in women over 65, it is not in young AN patients with prolonged amenorrhea.
\end{abstract}

Keywords: Anorexia Nervosa; Amenorrhea; Osteoporosis; Fracturerisk; DXA; BMD

\section{INTRODUCTION}

Anorexia nervosa (AN) is a mental disorder whose features are deliberate weight loss, disordered body image, and intrusive overvalued fears of gaining weight.

Secondary amenorrhea (absence of at least 3 consecutive menstrual cycles or presence of menstrual cycles only following hormone administration) was one of the DSM-IV criteria for AN diagnosis [1] and it is a key feature of its possible medical complications.
Long-term consequences of AN include endocrine dysfunctions resulting in low levels of gonadotropins and estrogens, secondary amenorrhea, bone loss and/or osteoporosis [2-4]. Bone reabsorption is related with the exposure to low levels of estrogens, leading to an increased risk of bone fractures as it happens in late postmenopausal period [5]. According to recent studies among postmenopausal women aged 50 years or older $39.6 \%$ of patients had osteopenia ( $\mathrm{T}$ score of -1 to -2.49 ) and $7.2 \%$ had osteoporosis ( $\mathrm{T}$ score $</=-2.5$ ). Osteoporosis was associated with a fracture rate approximately 4 times that of normal BMD (rate ratio, 4.03; 95\% confidence interval $[\mathrm{CI}], 3.59-4.53)$ and osteopenia was associated with a 1.8 -fold higher rate $(95 \% \mathrm{CI}, 1.49$ - 2.18$)$; moreover peripheral BMD results and years of age were highly predictive of fracture risk [6-8].

AN frequently has its onset during adolescence [9-11], when peak bone mass is normally reached, and an anorectic episode in youth may permanently impair skeletal integrity and lead to debilitating fractures [12-14]. Therefore young women with AN may develop a risk for fractures comparable to that of postmenopausal women (Figure 1). As osteoporosis is widely accepted as a "female disease" occurring primarily in postmenopausal women, the fact that this disease may affect premenopausal women experiencing menstrual dysfunction is less commonly known $[15,16]$. Patients with AN usually require hospitalization when their weight falls below $70 \%$ of ideal body weight. Accordingly, patients hospitalized with severe AN tend to suffer from many starvation-related clinical and biochemical abnormalities [17]. Among these starvation-related medical complications endocrine-based bone loss may put AN patients at risk for pathological fractures $[18,19]$. The purpose of this case report series was to assess the influence of eating disorder related amenorrhea on bone mineral density (BMD) in a sample of women hospitalized for AN. 

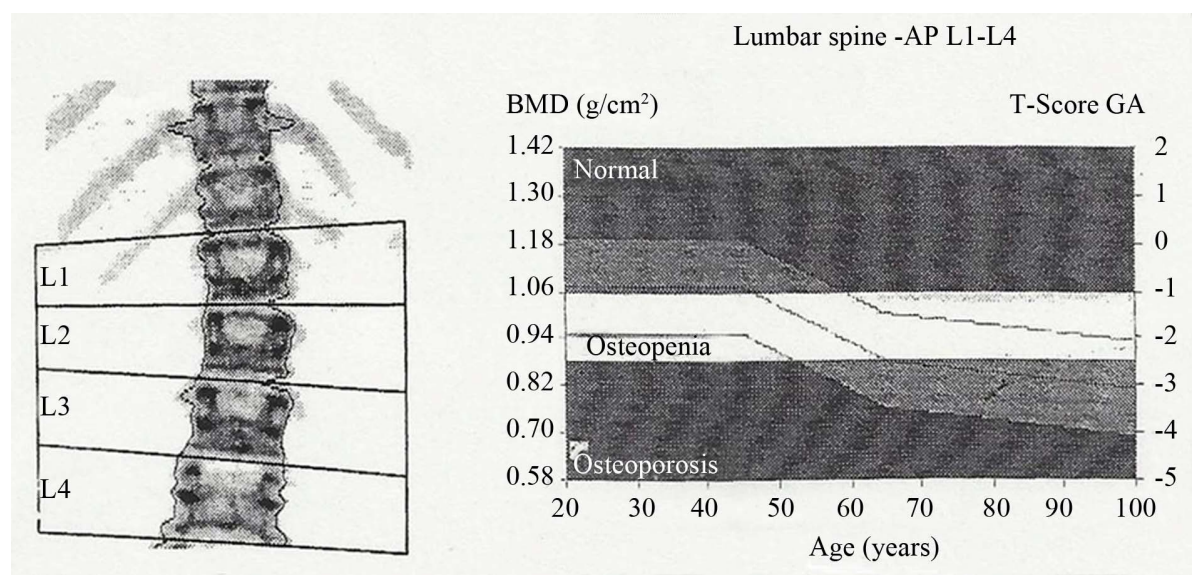

Figure 1. Osteoporosis in a 22-year-old patient with AN.

\section{METHODS}

Weevaluated BMD by Dual energy X-ray Absorptiometry (DXA) in 19patientshospitalizedat the Psychiatric Clinic of University of Pisa between March 2008 and July 2011 because of severe AN.

We used Open Office 3.3 Calc software for data management.

During our observation patients undergone a full medical evaluation as for possible complications of $\mathrm{AN}$, with special focus on endocrine and metabolic dysfunctions affecting bone structure. BMD, along with the potential risk of pathological fractures, were specifically assessed by DXA.

DXA uses X-rays to assess BMD by measuring different X-ray absorption proportional to the density of bone tissue traversed. DXA is considered the gold standard for the diagnosis of osteoporosis. Osteoporosis is diagnosed when BMD is less than or equal to 2.5 standard deviations below that of a young adult reference population. Standard deviation units used in relation to the young adult healthy population are called T-scores.

The World Health Organization (WHO) has established the following diagnostic criteria:

- T-Score -1.0 or greater is considered "normal";

- T-Score between -1.0 and -2.5 is "osteopenia", and is associated with "increased fracture risk";

- T-Score -2.5 or aboveis "osteoporosis", and is associated with "high fracture risk".

\section{RESULTS}

All subjects in our sample were females, their age ranged between 18 and 50 years (average $30.63 \pm 9.3$ ) and their average body weight was $39.16 \pm 7.87 \mathrm{~kg}$ (from 26 to 58), with a Body Mass Index (BMI) being among 11.1 and $17.9 \mathrm{~kg} / \mathrm{m}^{2}$ (average $14.58 \pm 1.87$ ). All of the patients suffered from prolonged amenorrhea with a mean duration of $3.6 \pm 3.1$ months (from 0.3 to 12 ) and only 8 patients out of 19 were taking hormone replacement therapy (HRT) at the beginning of our observation. After DXA assessment all of the patients showed a lumbar/femoral bone loss or osteoporosis with an increased fracture risk in comparison with peers and comparable to that of postmenopausal women.

The average $\mathrm{T}$ Score for lumbar spine was -2.27 ( \pm $0.98)$ and average $\mathrm{T}$ Score for femur neck was -1.86 ( \pm 0.84 ) with only one patient having no lumbar spine fracture risk increased, while in the rest of the sample lumbar spine fracture risk was considered "increased" or "high". Similarly, but less severely, only two patients had femur neck fracture risk not increased, while in the other patients femur neck fracture risk was considered "increased" or "high" according to World Health Organization (WHO) standards (Table 1).

\section{DISCUSSION}

Although expected it's striking to find such an elevated fracture risk in a sample of women whose age range is between 18 and 50 years of age, and it's noteworthy that only a minority of patients with such a severe risk of medical complications were taking hormone replacement therapy. However this is in line with a part of the literature data suggesting that clinicians tend to underestimate the risks related with bone loss in this specific population of patients [20-22].

Preliminary data from our observation supports that low levels of estrogens, related with AN psychopathology, do have an impact on BMD; with increasing concern about the likelihood of pathological fractures in a population of young people.

As a consequence young patients affected by AN may have a risk of pathological fractures comparable to that of postmenopausal women of more than 50 years of age.

Women under 50 years of age with AN and prolonged amenorrhea are not routinely assessed as for BMD and fracture risk, while postmenopausal women over 65 
Table 1. Bone mineral density in AN patients.

\begin{tabular}{|c|c|c|c|c|c|c|c|c|c|}
\hline \multirow{2}{*}{ Patient \# } & \multirow{2}{*}{ Age } & \multirow{2}{*}{$\begin{array}{c}\text { Body weight } \\
(\mathrm{kg})\end{array}$} & \multirow{2}{*}{$\begin{array}{l}\mathrm{BMI}^{\mathrm{a}} \\
\left(\mathrm{kg} / \mathrm{m}^{2}\right)\end{array}$} & \multicolumn{2}{|c|}{ T-Score } & \multicolumn{2}{|c|}{ Fracture risk } & \multirow{2}{*}{$\begin{array}{l}\mathrm{LPA}^{\mathrm{b}} \\
\text { (years) }\end{array}$} & \multirow{2}{*}{$\mathrm{HRT}^{\mathrm{C}}$} \\
\hline & & & & Lumbar spine & Femur neck & Lumbar spine & Femur neck & & \\
\hline 1 & 34 & 31 & 12.9 & -2.7 & -3 & high & high & 4 & no \\
\hline 2 & 44 & 46 & 17.9 & -2.2 & -3.3 & increased & high & 6 & yes \\
\hline 3 & 25 & 34 & 12.8 & -3.1 & -1.4 & high & increased & 2 & no \\
\hline 4 & 20 & 33 & 11.9 & -2.7 & -1.5 & high & increased & 1 & no \\
\hline 5 & 24 & 36 & 12.7 & -2.3 & -1.3 & high & increased & 8 & no \\
\hline 6 & 27 & 43 & 16.2 & -1.7 & -2 & increased & high & 0,5 & no \\
\hline 7 & 50 & 58 & 14.8 & -3.5 & -1.5 & increased & increased & 8 & no \\
\hline 8 & 18 & 32 & 14.6 & -2.4 & -1.2 & increased & increased & 3 & no \\
\hline 9 & 22 & 26 & 11.1 & -3.1 & -3.2 & high & high & 5 & yes \\
\hline 10 & 23 & 48 & 16.2 & -0.7 & -1.8 & normal & increased & 1 & yes \\
\hline 11 & 43 & 38 & 17.1 & -2.7 & -2.6 & high & high & 3 & no \\
\hline 12 & 29 & 37 & 13.8 & -2.5 & -1.5 & high & high & 1 & yes \\
\hline 13 & 27 & 51 & 17.2 & -0.8 & -0.5 & normal & normal & 0,3 & yes \\
\hline 14 & 37 & 46 & 15 & -1.4 & -0.7 & increased & normal & 1 & no \\
\hline 15 & 30 & 39 & 14.7 & -3.2 & -2.3 & high & high & 3 & yes \\
\hline 16 & 30 & 37 & 13.4 & -4.1 & -2.6 & high & high & 12 & yes \\
\hline 17 & 20 & 32 & 14.2 & -1.9 & -0.9 & increased & normal & 2 & no \\
\hline 18 & 36 & 37 & 14.4 & -1.8 & -1.4 & increased & increased & 1 & no \\
\hline 19 & 43 & 40 & 16.2 & -0.4 & -2.7 & normal & high & 2 & yes \\
\hline
\end{tabular}

${ }^{a}$ BMI, Body Mass Index; ${ }^{b}$ LPA, Longest Period of Amenorrhea; ${ }^{\circ}$ HRT, Hormonal Replacement Therapy.

years of age are [23]. This is particularly significant because, contrary to what happens in postmenopausal women, osteoporosis in young AN patients is a predictable, measurable, manageable and potentially reversible condition. Within this framework a careful assessment of BMD by DXA in AN patients with prolonged amenorrhea may contribute to the prevention of the consequences of this disorder.

Some limitations of this work must be acknowledged. First, the small number of patients does not allow to draw conclusions about the risks related with bone loss in AN. Second, all of the patients assessed as for BMD were hospitalized, and the severity of AN may potentially bias our sample.

\section{CONCLUSION}

$\mathrm{AN}$ is a psychiatric disorder resulting in medical complications and the DXA assessment did not demonstrate a simple osteopenia, but an osteoporosis with significant fracture risk in a group of relatively young people. This datum cannot be ignored, even though the assessment of the actual risk needs further studies in larger samples to solve the questions about bone loss and fracture risk in young patients with AN.

\section{REFERENCES}

[1] American Psychiatric Association (1994) Diagnostic and statistical manual of mental disorders. 4th Edition, Washington DC.

[2] Lawson, E.A. and Klibanski, A. (2008) Endocrine abnormalities in anorexia nervosa. Nature Clinical Practice Endocrinology \& Metabolism, 4, 407-414. http://dx.doi.org/10.1038/ncpendmet 0872

[3] Mehler, P.S., Cleary, B.S. and Gaudiani, J.L. (2011) Osteoporosis in anorexia nervosa. Eating Disorders, 19, 194-202. http://dx.doi.org/10.1080/10640266.2011.551636

[4] Salisbury, J.J. and Mitchell, J.E. (1991) Bone mineral density and anorexia nervosa in women. American Journal of Psychiatry, 148, 768-774.

[5] Kanis, J.A. (1994) Assessment of fracture risk and its application to screening for postmenopausal osteoporosis: 
Synopsis of a WHO report. WHO Study Group. Osteoporosis International, 4, 368-381. http://dx.doi.org/10.1007/BF01622200

[6] Siris, E.S., Miller, P.D., Barrett-Connor, E., Faulkner, K.G., Wehren, L.E., Abbott, T.A., et al. (2001) Identification and fracture outcomes of undiagnosed low bone mineral density in postmenopausal women: Results from the National Osteoporosis Risk Assessment. Journal of the American Medical Association, 286, 2815-2822. http://dx.doi.org/10.1001/jama.286.22.2815

[7] Siris, E.S., Brenneman,S.K., Barrett-Connor, E., Miller, P.D., Sajjan, S., Berger, M.L. and Chen, Y.T. (2006) The effect of age and bone mineral density on the absolute, excess, and relative risk of fracture in postmenopausal women aged 50-99: Results from the National Osteoporosis Risk Assessment (NORA). Osteoporosis International, 17, 565-574. http://dx.doi.org/10.1007/s00198-005-0027-4

[8] Siris, E.S., Brenneman, S.K., Miller, P.D., Barrett-Connor, E., Chen, Y.T., Sherwood, L.M. and Abbott, T.A. (2004) Predictive value of low BMD for 1-year fracture outcomes is similar for postmenopausal women ages 5064 and 65 and Older: Results from the National Osteoporosis Risk Assessment (NORA). Journal of Bone and Mineral Research, 19, 1215-1220. http://dx.doi.org/10.1359/JBMR.040508

[9] Favaro, A., Caregaro, L., Tenconi, E., Bosello, R. and Santonastaso, P. (2009) Time trends in age at onset of anorexia nervosa and bulimia nervosa. Journal of Clinical Psychiatry, 70, 1715-1721. http://dx.doi.org/10.4088/JCP.09m05176blu

[10] Abbate-Daga, G., Pierò, A., Rigardetto, R., Gandione, M., Gramaglia, C. and Fassino, S. (2007) Clinical, psychological and personality features related to age of onset of anorexia nervosa. Psychopathology, 40, 261-268. http://dx.doi.org/10.1159/000101731

[11] Grange, D. and Loeb, K.L. (2007) Early identification and treatment of eating disorders: Prodrome to syndrome. Early Intervention in Psychiatry, 1, 27-39. http://dx.doi.org/10.1111/j.1751-7893.2007.00007.x

[12] Wolfert, A. and Mehler, P.S. (2002) Osteoporosis: Prevention and treatment in anorexia nervosa. Eating and Weight Disorders, 7, 72-81.

[13] Misra, M. and Klibanski, A. (2002) Evaluation and treatment of low bone density in anorexia nervosa. Nutrition in Clinical Care, 5, 298-308. http://dx.doi.org/10.1046/j.1523-5408.2002.05605.x

[14] Misra, M. and Klibanski, A. (2011) Bone metabolism in adolescents with anorexia nervosa. Journal of Endocrine Investigations, 34, 324-332.

[15] McGee, C. (1997) Secondary amenorrhea leading to osteoporosis: Incidence and prevention. Nurse Practicioner, 22, 38-48. http://dx.doi.org/10.1097/00006205-199705000-00004

[16] Legroux-Gérot, I., Vignau, J., D’Herbomez, M., Collier, F., Marchandise, X., Duquesnoy, B., et al. (2007) Evaluation of bone loss and its mechanisms in anorexia nervosa. Calcified Tissue International, 81, 174-182. http://dx.doi.org/10.1007/s00223-007-9038-9

[17] Gaudiani, J.L., Kashuk, J.L., Chu, E.S., Narayanan, V. and Mehler, P.S. (2010) The use of thrombelastography to determine coagulation status in severe anorexia nervosa: A case series. International Journal of Eating Disorder, 43, 382-385.

[18] Misra, M. and Klibanski, A. (2006) Anorexia nervosa and osteoporosis. Reviews in Endocrine and Metabolic Disorders, 7, 91-99. http://dx.doi.org/10.1007/s11154-006-9005-1

[19] Misra, M. and Klibanski, A. (2011) Bone health in anorexia nervosa. Current Opinion in Endocrinology, Diabetes and Obesity, 18, 376-382. http://dx.doi.org/10.1097/MED.0b013e32834b4bdc

[20] Misra, M. and Klibanski, A. (2010) Neuroendocrine consequences of anorexia nervosa in adolescents. Endocrine Development, 17, 197-214. http://dx.doi.org/10.1159/000262540

[21] Teng, K. (2011) Premenopausal osteoporosis, an overlooked consequence of anorexia nervosa. Cleveland Clinical Journal of Medicine, 78, 50-58. http://dx.doi.org/10.3949/ccjm.78a.10023

[22] Fernández Soto, M.L., González Jiménez, A. and Varsavsky, M. (2010) Bone metabolism and fracture risk in anorexia nervosa. Medicina Clínica (Barcelona), 135, $274-$ 279. http://dx.doi.org/10.1016/j.medcli.2009.04.046

[23] Gourlay, M.L., Fine, J.P., Preisser, J.S., May, R.C., Li, C., Lui, L.Y., et al. (2012) Study of Osteoporotic Fractures Research Group. Bone-density testing interval and transition to osteoporosis in older women. New England Journal of Medicine, 366, 225-233. http://dx.doi.org/10.1056/NEJMoa1107142 